\title{
Ansiedad, depresión, estrés y organización de la personalidad en estudiantes novatos de medicina y enfermería
}

\section{Anxiety, depression, stress and personality organization in medical and nursing freshmen}

René Barraza L. ,a, Nadia Muñoz N. 2,b, Marco Alfaro G. ${ }^{3, c}$, Álvaro Álvarez M. 1,d, Valeria Araya T. ${ }^{1, d}$, Johan Villagra C. ${ }^{1, d}$ y Ana María Contreras A.,

\begin{abstract}
Introduction: The health students are likely to experience anxiety, depression and stress freshman year, affecting their performance and adaptation. The scant evidence of the relationship between personality like dispositional-condition with the above symptoms, limited understanding of this phenomenon in the studied sample. Objective: To establish the relationship of the dimensions of personality, anxiety, depression and stress freshmen in medical and nursing. Method: We surveyed the Kernberg inventory of personality organization and scale of depression, anxiety and stress, to a sample of 110 university/as first-year medical and nursing schools, three universities of La Serena and Coquimbo, data thrown by two instruments were correlated using $r$ Pearson's. Results: It was found correlation between all dimensions of personality organization, with anxiety, depression and stress. In addition, $47 \%$ have anxiety, depression $28 \%$ and $44 \%$ stress amid a severe ranges. A 65\% rate with borderline personality structure where two dimensions of personality have high values. Conclusion: It is found that the greater integration of the personality, the lower the level of anxiety, depression and stress, Since it is proposed to evaluate early both conditions would guide remedial actions that favor the development of these students early stages of the learning process moreover the discussion arises around being considered to evaluate behaviors entries in the selection process to the races.
\end{abstract}

Key words: Personality, anxiety, depression, stress, Health Student. Rev Chil Neuro-Psiquiat 2015; 53 (4): 251-260

Recibido: 14/09/2015

Aceptado: 28/10/2015

Los autores no presentan ningún tipo de conflicto de interés.

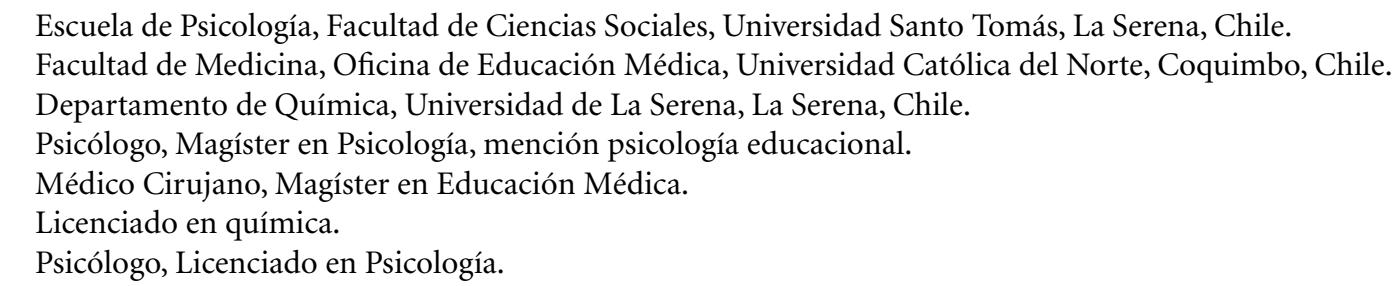




\section{Introducción}

$\mathrm{L}$ os retos relacionados con la vida independiente y los desafíos académicos que comúnmente enfrentan los estudiantes universitarios, generan una mayor potencial predisposición a la emergencia de depresión, ansiedad y estrés ${ }^{1-4}$. De acuerdo a la literatura disponible, la prevalencia de estos cuadros es mayor en carreras del área de salud si se le compara con otros dominios disciplinares ${ }^{5-9}$, alcanzando incluso mayores niveles que la población general ${ }^{10}$. Esta condición se vincularía a los niveles de exigencia académica propios de las carreras de salud, factores socioeconómicos y socioemocionales ${ }^{10,11}$, además de variables como el tipo de carrera, el sexo $^{4}$, y ser alumno/a de primer año en la carrera ${ }^{11}$.

Un aspecto a considerar en cuanto a la emergencia de estos cuadros, es la relación de ellos con la personalidad de los estudiantes de la salud ${ }^{12-16}$. En la actualidad los estudios que vinculan estas variables, son escasos y toman como referencia teórica el modelo de los cinco grandes factores ${ }^{17}$. Esta aproximación ha sido criticada por centrarse en la descripción de rasgos ${ }^{18,19}$, lo cual condiciona su potencial explicativo respecto a la génesis de la depresión, ansiedad y estrés en este grupo de universitarios ${ }^{20}$.

En este sentido, el modelo de personalidad propuesto por Kernberg ${ }^{18-20}$ permite abordar estas críticas, al proponer que la personalidad se organiza en base a criterios dimensionales, los cuales se forman a partir del temperamento que es definido como condiciones disposicionales genéticas e innatas de escasa modificabilidad. Estas condiciones disposicionales, modulan la intensidad, ritmo y umbral de activación afectiva, impactando en el propio sujeto y su relación con los demás. La presencia o ausencia de estos criterios dimensionales, da como resultado el establecimiento de patrones estructurales de personalidad reconocibles, más o menos rígidos, que le permiten al individuo conducirse en el mundo con mayor o menor eficiencia emocional y adaptativa. De esta forma, mientras más limitado sea el funcionamiento de estos criterios dimensionales, mayor será la propensión al desencadenamiento de afectos negativos intensos, ejerciéndose un influjo permanente en el bienestar emocional del individuo ${ }^{21}$.

Lo anterior es relevante si se considera que la personalidad -conceptualizada desde la influencia ejercida por las condiciones disposicionales como el temperamento-, presenta escasa evidencia empírica de relación con los síntomas antes descritos ${ }^{22}$, limitando la comprensión de este fenómeno, más aún en el grupo al cual el presente estudio buscó abordar. Esto puede ser una cuestión de suyo crítica, si se considera que la mayor parte de los estudiantes de primer año de universidad, se encuentra en tránsito de la adolescencia a la adultez, período en el cual debe producirse la consolidación de la identidad y por ende la cristalización de la personalidad, a partir de la integración y calidad de funcionamiento de estas dimensiones ${ }^{23,24}$.

Por otra parte, la clarificación de esta relación permitiría reconocer aspectos que se vinculan a cuestiones incidentes en el retraso y fracaso académico, como también al abandono temprano de la carrera $^{4,10,11}$. Dados estos antecedentes, el presente estudio buscó establecer el grado de relación asociativa de las dimensiones de organización de la personalidad con los síntomas depresivos, ansiosos y estrés, en una muestra de estudiantes universitarios de carreras del área de la salud de primer año, en tres universidades de La Serena y Coquimbo.

\section{Materiales y Método}

\section{Participantes}

El presente estudio corresponde a un diseño no experimental, de tipo transeccional de carácter correlacional $^{25,26}$, realizado en una muestra de 110 estudiantes de primer año de la cohorte 2013, cuyas edades fluctuaban entre los 18 a 34 años $(M=20,7$; $\mathrm{DE}=3,41)$ y su moda es de 18 , correspondientes a las carreras de medicina (66 estudiantes) y enfermería (44 estudiantes), de tres universidades de la Serena y Coquimbo, IV Región de Coquimbo, Chile.

\section{Procedimientos}

Previa aprobación del comité de ética de la universidad patrocinante, se procedió a seleccionar a la 
muestra mediante un procedimiento de muestreo no probabilístico de tipo voluntario ${ }^{26}$, donde de un total de 70 alumnos/as de medicina sólo 4 declinaron participar, y en enfermería de un total de 47 sólo 3 declinaron participar, alcanzando un nivel de participación de un $94,2 \%$ en medicina y de un 93,6 en enfermería. Se coordinó con los jefes de carrera de las distintas universidades la fecha y hora adecuada para presentar el proyecto a los estudiantes, se explicó a los diferentes cursos los objetivos, términos referidos a la confidencialidad de las respuestas y compromiso de entrega de resultados individualizados a quienes desearan conocerlos, para finalmente proceder a la firma del consentimiento informado a quienes voluntariamente participaron del estudio. Una vez realizado todo lo anterior, se procedió a la aplicación de los instrumentos. Para el reporte de resultados individuales fue necesario solicitar datos de identificación de los participantes e informar las vías de contacto con el investigador principal, pese a ello ninguno de los participantes solicitó posterior a la evaluación dicha información.

\section{Instrumentos}

Las dimensiones de la organización de la personalidad fueron evaluadas a través del Inventario de Organización de la Personalidad (IPO), versión adaptada y validada para Chile ${ }^{27,28}$. El cual es un inventario de autorreporte que se compone de 83 reactivos organizados en cinco escalas tipo lickert: tres primarias (difusión de identidad, uso de defensas primitivas, examen de realidad) y dos globales (agresión y valores morales). Este instrumento ha sido aplicado en población universitaria ${ }^{29}$, pero no reporta aplicaciones previas específicamente en la población de estudio. Pese a ello, en la presente aplicación alcanzó una confiabilidad total de 0,92 mediante alfa de Cronbach, en tanto que las escalas primarias obtuvieron coeficientes alfa de 0,80 para defensas primitivas, 0,82 para difusión de identidad y 0,87 para examen de realidad, las escalas globales alcanzaron coeficientes alfa de 0,74 y 0,73 , para valores morales y agresión respectivamente. Esto permite afirmar que los datos obtenidos son confiables. Por otra parte, reporta una adecuada validez en población chilena ${ }^{28}$.
En cuanto al reporte de ansiedad, depresión y estrés se utilizó la Escala de Depresión, Ansiedad y Estrés (DASS-21), versión adaptada y validada para Chile $^{30}$. Esta escala de autorreporte que se compone de 21 reactivos organizados en tres escalas tipo lickert: 7 ítems para depresión, 7 para ansiedad y 7 para estrés. De acuerdo a la literatura este instrumento ha sido aplicado en estudiantes del área de la salud ${ }^{2,4,7}$. En la presente medición la escala alcanzó una confiabilidad total de 0,80 mediante alfa de Cronbach, determinándose un coeficiente alfa de 0,70 para ansiedad, 0,82 para depresión y 0,80 para estrés, lo cual es concordante con otras aplicaciones en poblaciones de universitarios ${ }^{4-6}$. Este instrumento ha presentado buenos indicadores de validez en población chilena universitaria ${ }^{29}$.

Los datos obtenidos fueron procesados con el paquete estadístico SPSS 15, donde se trabajó con medidas de tendencia central para la descripción, t de Student para la comparación de la ansiedad, depresión y estrés por carrera y sexo, y el coeficiente de correlación $\mathrm{r}$ de Pearson para el análisis correlacional.

\section{Resultados}

Respecto a la sintomatología ansiosa, depresiva y estrés es posible constatar que para la ansiedad un 53\% de la muestra se sitúa en rango normal, un $35 \%$ en rango medio a moderado y un $12 \%$ en rango severo a muy severo. En cuanto a la depresión un $72 \%$ de la muestra se sitúa en rango normal, un $22,4 \%$ en rango medio a moderado y un $5,6 \%$ en rangos severo a muy severo, finalmente para el estrés un $56 \%$ de la muestra se sitúa en rango normal, un $32 \%$ en rango medio a moderado y un $12 \%$ en rangos severo a muy severo. No obstante los puntajes promedio sitúan a la muestra total en rangos normales para ansiedad y depresión y en el caso del estrés en rango medio (Tabla 1).

Si se observa el comportamiento de las medias por carreras se evidencia que la única sub-escala en donde todas las carreras puntúan dentro de los puntajes de normalidad es depresión, la sub-escala de ansiedad reporta puntajes sobre lo esperado 
en medicina, situándolo en un rango medio, en tanto que la sub-escala de estrés ubica a medicina y enfermería en rango medio. En cuanto a la comparación de estos promedios por carrera, sólo se encontraron diferencias significativas en los puntajes promedio de depresión (Tabla 2) y en cuanto a la comparación por sexo no se encontraron diferencias significativas entre hombres y mujeres para estas variables (Tabla 3 ).

Respecto a la caracterización de la personalidad,

Tabla 1. Media y desviación estándar de la muestra total de estudiantes de salud, para las ansiedad, depresión y estrés

\begin{tabular}{|lccccc|}
\hline & n & Mínimo & Máximo & Media & $\begin{array}{c}\text { Desv. } \\
\text { típ. }\end{array}$ \\
\hline Ansiedad & 110 & 0 & 30 & 7,64 & 6,576 \\
Depresión & 110 & 0 & 30 & 6,73 & 6,358 \\
Estrés & 110 & 0 & 38 & 15,40 & 8,007 \\
\hline
\end{tabular}

el 55,04\% de la muestra correspondía a una organización "Límite Alta" (61 sujetos), un 5,504\% a una organización "Limite Baja" (6 sujetos) y un 39,44\% a una organización "Neurótica" (43 sujetos). Respecto a los puntajes obtenidos por la muestra total en las dimensiones de la personalidad, se aprecia que la dimensión uso de defensas primitivas y valores morales se encuentran por sobre valores esperados para muestras no clínicas.

Si se observa el comportamiento de las medias de las dimensiones de organización de la personalidad por carreras, se evidencia que uso de defensas primitivas $(33,820 \pm 8,594)$ y valores morales $(22,176 \pm 6,253)$ describen puntajes promedio tanto en medicina como en enfermería, por sobre lo esperado para muestras no clínicas ${ }^{27}$ (Tabla 4).

Finalmente, en cuanto a la correlación de las variables, fue posible establecer que todas dimensiones de organización de la personalidad presentan correlaciones directas significativas con ansiedad, depresión y estrés (Tabla 5).

Tabla 2. Descripción y comparación por carrera, para las ansiedad, depresión y estrés

\begin{tabular}{|c|c|c|c|c|c|c|c|c|}
\hline & & $\mathbf{n}$ & Media & Des. típica & $\mathbf{t}$ & Gl & Sig. (bilateral) & Diferencia de medias \\
\hline Ansiedad & $\begin{array}{l}\text { Med } \\
\text { Enf }\end{array}$ & $\begin{array}{l}66 \\
44\end{array}$ & $\begin{array}{l}8,03 \\
7,05\end{array}$ & $\begin{array}{l}7,064 \\
5,795\end{array}$ & 0,799 & 103,322 & 0,426 & 0,985 \\
\hline Depresión & $\begin{array}{l}\text { Med } \\
\text { Enf }\end{array}$ & $\begin{array}{l}66 \\
44\end{array}$ & $\begin{array}{l}7,67 \\
5,32\end{array}$ & $\begin{array}{l}7,389 \\
4,068\end{array}$ & 2,141 & 104,795 & $0,035^{*}$ & 2,348 \\
\hline Estrés & $\begin{array}{l}\text { Med } \\
\text { Enf }\end{array}$ & $\begin{array}{l}66 \\
44\end{array}$ & $\begin{array}{l}15,70 \\
14,95\end{array}$ & $\begin{array}{l}8,674 \\
6,962\end{array}$ & 0,475 & 108 & 0,636 & 0,742 \\
\hline
\end{tabular}

${ }^{\star}$ La diferencia de medias es significativa al nivel 0,05.

Tabla 3. Descripción y comparación por sexo, para las ansiedad, depresión y estrés

\begin{tabular}{|llccccccc|}
\hline \multirow{2}{*}{ Ansiedad } & Sexo & $\mathbf{n}$ & Media & Des. típica & $\mathbf{t}$ & gl & Sig. (bilateral) & Diferencia de medias \\
& Hombre & 45 & 8,00 & 7,580 & 0,481 & 108 & 0,632 & 0,615 \\
& Mujer & 65 & 7,38 & 5,830 & & & & 0,725 \\
\multirow{2}{*}{ Depresión } & Hombre & 45 & 7,16 & 7,217 & 0,562 & 80,251 & 0,576 & $-1,467$ \\
& Mujer & 65 & 6,43 & 5,728 & & & & \\
\multirow{2}{*}{ Estrés } & Hombre & 45 & 14,53 & 9,109 & $-0,944$ & 108 & 0,347 & \\
& Mujer & 65 & 16,00 & 7,159 & & & & \\
\hline
\end{tabular}

${ }^{\star}$ La diferencia de medias es significativa al nivel 0,05 . 


\section{Discusión}

En cuanto a la presencia de ansiedad, depresión y estrés, es posible constatar que los resultados obtenidos por la muestra se asemejan con los datos de prevalencia declarados en la literatura internacional para la ansiedad ${ }^{2}$ y el estrés ${ }^{6,7}$, siendo levemente más bajos en depresión que los reportados para muestras de universitarios de salud ${ }^{2,6}$. Por otra parte, si se comparara los resultados obtenidos con los de otros estudios hechos en Chile ${ }^{10,32-34}$, se constata que los puntajes alcanzados en la actual aplicación, son ampliamente superiores a lo reportado para universitarios chilenos ${ }^{4}$. Esto concuerda con lo señalado en la literatura, en relación a la mayor presencia de este tipo de sintomatología en

Tabla 4. Descripción de la muestra total de estudiantes de salud, para las dimensiones de organización de la personalidad

\begin{tabular}{|lccccc|}
\hline Dimensión & $\mathbf{n}$ & Mínimo & Máximo & Media & $\begin{array}{c}\text { Desv. } \\
\text { típ. }\end{array}$ \\
\hline Defensas & 110 & 25 & 66 & 43,00 & 9,241 \\
Identidad & 110 & 22 & 72 & 45,11 & 10,141 \\
Realidad & 110 & 20 & 62 & 33,67 & 8,892 \\
V. Morales & 110 & 20 & 73 & 42,43 & 10,116 \\
Agresión & 110 & 20 & 60 & 26,62 & 6,008 \\
\hline
\end{tabular}

carreras del área de la salud respecto de otras carreras universitarias ${ }^{5-9}$. No obstante, los resultados de esta comparación deben ser interpretados con precaución, dada la diversidad de instrumentos de medición utilizados en los diversos estudios. Pese a ello, igualmente es interesante el hecho que distintas mediciones de este fenómeno arrojan resultados cualitativamente similares, lo cual puede estar dando cuenta de un patrón susceptible de observar.

En cuanto a la comparación por carrera, la constatación de diferencias significativas en la escala de depresión a favor de medicina, no debiera significar una cuestión de cuidado, toda vez que los puntajes de dicha escala se situaron en niveles de normalidad. No obstante y atendiendo a lo propuesto en la literatura internacional ${ }^{2,6,7}$, se propone la necesidad de tomar medidas preventivas a fin de evitar que los estudiantes de medicina transiten a niveles de deterioro en esta variable, lo cual afecta de manera directa su desempeño y aprendizaje en los niveles iniciales de la carrera ${ }^{9}$.

Por otra parte, en lo referido a la comparación por sexo la no presencia de diferencias entre hombres y mujeres, es contraria a lo reportado en la literatura que señala mayor presencia de ansiedad y estrés en las mujeres ${ }^{2,4}$. Esta condición de indiferenciación por sexo encontrada en el grupo estudiado, podría considerarse como un aspecto facilitador para la planificación y ejecu-

Tabla 5. Correlación entre dimensiones de organización de la personalidad y ansiedad, depresión y estrés

\begin{tabular}{|llccc|}
\hline Dimensión & Sig & Ansiedad & Depresión & Estrés \\
Defensas & r de Pearson & $0,340^{* *}$ & $0,373^{* *}$ & $0,304^{* *}$ \\
& Sig. (bilateral) & 0,000 & 0,000 & 0,001 \\
Identidad & r de Pearson & $0,444^{* *}$ & $0,370^{* *}$ & $0,343^{* *}$ \\
& Sig. (bilateral) & 0,000 & 0,000 & 0,000 \\
Realidad & r de Pearson & $0,360^{\star *}$ & $0,307^{\star *}$ & $0,269^{* *}$ \\
& Sig. (bilateral) & 0,000 & 0,001 & 0,004 \\
V. Morales & r de Pearson & $0,307^{* *}$ & $0,283^{* *}$ & $0,326^{* *}$ \\
& Sig. (bilateral) & 0,001 & 0,003 & 0,001 \\
Agresión & r de Pearson & $0,206^{*}$ & $0,188^{*}$ & $0,244^{*}$ \\
& Sig. (bilateral) & 0,031 & 0,049 & 0,010 \\
\hline
\end{tabular}

${ }^{\star}$ La correlación es significante al nivel 0,05 (bilateral). ${ }^{\star \star}$ La correlación es significativa al nivel 0,01 (bilateral). 
ción de acciones preventivas de orden académico -como entrenamiento en estrategias de estudio $u$ optimización en el uso del tiempo- y otras extracurriculares -como talleres de manejo del estrés y desarrollo de redes de apoyo social-, las cuales no requerirían de intervenciones separadas para hombres y mujeres, permitiendo la optimización de los habitualmente insuficientes recursos con que se cuenta en educación superior para el abordaje de este tipo de cuestiones.

En cuanto a la caracterización de la personalidad de los participantes, resulta interesante constatar el alto porcentaje de organización límite encontrado en el grupo estudiado. Esto pudiera tener relación con puntuaciones por sobre la media para poblaciones no clínicas ${ }^{29}$ en al menos dos de las cinco dimensiones evaluadas, a saber; uso de defensas primitivas y valores morales. En este sentido, se propone que el uso de defensas primitivas es un recurso de menor eficiencia adaptativa, lo cual genera mayores niveles de angustia y deterioro de las funciones yoicas utilizadas para la adaptación, flexibilidad y autonomía ${ }^{20}$. En tanto que los conflictos con la moral, la ética o los valores suelen experimentarse con altos niveles de angustia, ansiedad o depresión ${ }^{36}$. No obstante lo anterior, la interpretación de estos resultados debe tener en cuenta que la mayoría de los participantes en el estudio corresponde a estudiantes de 18 años, lo cual implica que aún están en el tránsito desde la adolescencia a la juventud ${ }^{24,25}$, por lo que es esperable que persista cierto grado de inestabilidad identitaria propio de la adolescencia ${ }^{37}$, lo cual permitiría brindar un cierto marco explicativo al alto porcentaje de participantes tamizados con organizaciones de personalidad límite. En este mismo sentido, es menester clarificar que el presente estudio evaluó, sin pretensiones diagnósticas ${ }^{21,27}$ sino más bien de tamizaje, la organización estructural de la personalidad desde un marco conceptual psicodinámico, lo que en ningún caso debe confundirse con diagnóstico de trastorno de personalidad limítrofe, puesto que esto último excede los objetivos del presente estudio.

No obstante lo anterior, los aspectos de inestabilidad identitatria detectados permiten brindar un soporte a la correlación encontrada entre todas las dimensiones que organizan la personalidad con la ansiedad, depresión y estrés. Dicho de otra forma, fue posible constatar que a mayor integración de la personalidad menor es la ocurrencia de ansiedad, depresión y estrés en la muestra de estudiantes evaluados. Esta afirmación encuentra sustento también en lo teórico ${ }^{21}$, lo cual propone que la no consolidación de la identidad se vincula a baja tolerancia a la ansiedad y a un bajo control de impulsos, caracterizado por: inflexibilidad e inestabilidad del yo, predominio de los afectos negativos, incoherencia en la experiencia de uno mismo y los demás ${ }^{19}$, condiciones promotoras de conflicto en el ámbito interpersonal generadoras de angustia y estrés ${ }^{22}$. Por otra parte, la relación directa de las dimensiones de la personalidad con los síntomas antes descritos, concuerda con resultados de otros estudios análogos ${ }^{23}$ que proponen la relación de aspectos disposicionales de personalidad con la reactividad emocional. No obstante, esta comparación debe realizarse con cautela, puesto que si bien, dicho estudio evaluó aspectos disposicionales como el temperamento y el carácter, hasta el momento no ha sido posible encontrar otros estudios que evidencien de manera específica la vinculación empírica entre las dimensiones de la personalidad descritas por Kernberg con la ansiedad, depresión y el estrés.

Finalmente, las correlaciones obtenidas permitieron responder al objetivo que buscó establecer la vinculación entre aspectos disposicionales de la personalidad, la ansiedad, la depresión y el estrés, en los estudiantes de las universidades que conformaron la muestra. Pese a que los resultados no son generalizables a otras escuelas de salud, resulta interesante constatar la presencia de esta relación, ya que tanto la personalidad como los síntomas anímicos, ansiosos y estrés pueden ser cuestiones interesantes de tamizar en etapas iniciales del proceso de formación en carreras de salud, a fin de orientar acciones potencialmente paliativas de estas condiciones ${ }^{8}$ que dificultan el desarrollo del estudiante en las mismas; por otra parte, pueden constituirse en aspectos susceptibles de ser evaluados como conductas de entradas en los procesos de selección a las carreras pudieran implementar ${ }^{37}$, a 
fin de facilitar el tránsito académico y personal de los estudiantes por el período de tiempo que dure la carrera.

A partir de lo anterior, resulta recomendable la realización de nuevos estudios con muestras más numerosas y representativas, que no sólo entreguen evidencia de la relación entre estas variables, sino que se hagan cargo de la pregunta subyacente, en relación al peso potencial, y por ende explicativo, que pudiera tener la personalidad -como con- dición disposicional y escasamente modificable-, respecto al origen de la ansiedad, la depresión y el estrés en los estudiantes de carreras de la salud. Esto permitiría reconocer la real importancia de este aspecto en el adecuado desarrollo y tránsito personal y educativo que realiza el estudiante por estas carreras, altamente demandantes, facilitando la focalización de los esfuerzos de intervención educacional como el apoyo psicológico referido al proceso de formación del estudiantado.

\begin{abstract}
Resumen
Introducción: Los estudiantes de salud están proclives a presentar ansiedad, depresión y estrés en el primer año de carrera, afectándose su desempeño y adaptación. La escasa evidencia de la relación entre la personalidad-como condición disposicional-con los síntomas antes mencionados, limita la comprensión de este fenómeno en la muestra estudiada. Objetivo: Establecer la relación de las dimensiones de personalidad, con la ansiedad, depresión y estrés en estudiantes de primer año de medicina y enfermería. Método: Se encuestó con el inventario de organización de personalidad de Kernberg y la escala de depresión, ansiedad y estrés, a una muestra de 110 universitarios/as de primer año de medicina y enfermería, de tres universidades de La Serena y Coquimbo, los datos arrojados por ambos instrumentos se correlacionaron usando $r$ de Pearson. Resultados: Se encontró correlación entre todas las dimensiones de organización de la personalidad, con la ansiedad, depresión y estrés. Además, un 47\% presenta ansiedad, 28\% depresión y 44\% estrés, en rangos medio a muy severo. Un $65 \%$ califica con estructura de personalidad limitrofe donde dos dimensiones de personalidad presentan valores altos. Conclusión: Se constata que a mayor integración de la personalidad, menor es el nivel de ansiedad, depresión y estrés, A partir de ello se propone que evaluar tempranamente ambas condiciones permitiría orientar acciones paliativas que favorezcan el desarrollo de estos estudiantes en etapas iniciales del proceso formativo, por otra parte, se plantea la discusión en torno a ser consideradas conductas de entradas a evaluar en los procesos de selección a las carreras.
\end{abstract}

Palabras clave: Personalidad, ansiedad, depresión, estrés, estudiantes de salud.

\section{Referencias bibliográficas}

1. Baader MT, Rojas CC, Molina FJ, Gotelli VM, Álamo PC, Fierro FC, et al. Diagnóstico de la prevalencia de trastornos de la salud mental en estudiantes universitarios y los factores de riesgo emocionales asociados. Rev Chil Neuro-Psiquiat
2014; 52 (3): 167-76. Disponible en: http://www. scielo.cl/scielo.php?script=sci_arttext\&pid=S071792272014000300004\&lng=es. http://dx.doi. org/10.4067/S0717-92272014000300004 [citado el 6 de abril de 2015].

2. Shamsuddin K, Fadzil F, Wan Ismail W, Azhar S, Omar K, Azimah N, et al. Correlates of depres- 
sion, anxiety and stress among Malaysian university students. Asian J Psychiatr 2013; 6 (4): 318-23. Disponible en: doi: 10.1016/j.ajp.2013.01.014 [citado el 6 de abril de 2015].

3. Feldman L, Goncalves L, Chacón PG, Zaragoza J, Bagés N, De Pablo J. Relaciones entre estrés académico, apoyo social, salud mental y rendimiento académico en estudiantes universitarios venezolanos. Universitas Psychologica 2008; 7739-751. Disponible en: http://www.redalyc.org/articulo. oa?id=64770311 [citado el 6 de abril de 2015].

4. Antúnez Z, Vinet EV. Problemas de salud mental en estudiantes de una universidad regional chilena. Rev Med Chile 2013; 141 (2): 209-16. Disponible en: http://www.scielo. $\mathrm{cl} /$ scielo.php?script=sci_arttext\&pid=S003498872013000200010\&lng=es. http://dx.doi. org/10.4067/S0034-98872013000200010 [citado el 6 de abril de 2015].

5. Marty M, Lavín GM, Figueroa MM, Larraín de la CD, Cruz MC. Prevalencia de estrés en estudiantes del área de la salud de la Universidad de los Andes y su relación con enfermedades infecciosas. Rev Chil Neuro-Psiquiatr 2005; 43 (1): 25-32. Disponible en: http://www.scielo. $\mathrm{cl} /$ scielo.php?script $=$ sci_arttext\&pid=S071792272005000100004\&lng=es. http://dx.doi. org/10.4067/S0717-92272005000100004 [citado el 6 de abril de 2015].

6. Arrieta Vergara K, Díaz Cárdenas S, González Martínez F. Síntomas de depresión, ansiedad y estrés en estudiantes de odontología: prevalencia y factores relacionados. Revista Colombiana de Psiquiatría 2013; 2: 173-81. Disponible en: http:// www.redalyc.org/articulo.oa?id $=80629187004$ [citado el 6 de abril de 2015].

7. Yusoff MS, Abdul Rahim AF, Baba AA, Ismail SB, Mat Pa MN, Esa AR. Prevalence and associated factors of stress, anxiety and depression among prospective medical students. Asian J Psychiatr 2013; 2: 128-33. Disponible en: doi: 10.1016/j. ajp.2012.09.012 [citado el 6 de abril de 2015].

8. Yusoff MSB, Rahim AFA, Yaacob MJ. Prevalence and sources of stress among Universiti Sains Malaysia Medical Students. Malays J Med Sci 2010; 1: 30-7. Disponible en: http://www.ncbi.nlm.nih.
gov/pmc/articles/PMC3216143/ [citado el 6 de abril de 2015].

9. Yusoff MSB, Rahim AFA, Yaacob MJ. The prevalence of final year medical students with depressive symptoms and its contributing factors International Medical Journal 2011; 4: 305-9. Disponible en: http://connection.ebscohost.com/c/ articles/71093485/prevalence-final-year-medicalstudents-depressive-symptoms-contributingfactors [citado el 6 de abril de 2015].

10. Santander TJ, Romero SM, Hitschfeld AM, Zamora AV. Prevalencia de ansiedad y depresión entre los estudiantes de medicina de la Pontificia Universidad Católica de Chile. Rev Chil Neuro-Psiquiatr 2011; 1: 47-55. Disponible en: http://www. scielo.cl/scielo.php?script=sci_arttext\&pid=S071792272011000100006\&lng=es. http://dx.doi. org/10.4067/S0717-92272011000100006 [citado el 6 de abril de 2015].

11. Chandavarkar U, Azzam A, Mathews CA. Anxiety symptoms and perceived performance in medical students. Depress Anxiety 2007; 2: 103-11. Disponible en: http://www.ncbi.nlm.nih.gov/ pubmed/16845642 [citado el 6 de abril de 2015].

12. Song Y, Huang Y, Liu D, Kwan JS, Zhang F, Sham PC, et al. Depression in college: depressive symptoms and personality factors in Beijing and Hong Kong college freshmen. Compr Psychiatry 2008; 5: 496-502. Disponible en: http://www.ncbi.nlm. nih.gov/pubmed/18702936 [citado el 6 de abril de 2015].

13. Karreman A, Van Assen M, Bekker M. Intensity of positive and negative emotions: Explaining the association between personality and depressive symptoms. Personality and Individual Differences 2013; 2: 214-220. Disponible en: http://www.sciencedirect.com/science/article/pii/ S0191886912004308 [citado el 6 de abril de 2015].

14. Friborg O, Martinussen M, Kaiser S, Øvergård K, Rosenvinge J. Comorbidity of personality disorders in anxiety disorders: A meta-analysis of 30 years of research. J Affect Disord 2013; 2: 143-55. Disponible en: doi: 10.1016/j.jad.2012.07.004 [citado el 6 de abril de 2015].

15. González P, Castillo M. Coping with stress and its relationship with personality dimensions, 
anxiety, and depression. Procedia-Social and Behavioral Sciences 2010; 5: 1562-73. Disponible en: http://www.sciencedirect.com/science/article/pii/ S1877042810017003 [citado el 6 de abril de 2015].

16. Peng L, Zhang J, Li M, Li P, Zhang Y, Zuo X, et al. Negative life events and mental health of Chinese medical students: The effect of resilience, personality and social support. Psychiatry Res 2012; 1: 138-41. Disponible en: doi: 10.1016/j. psychres.2011.12.006 [citado el 6 de abril de 2015].

17. Laverdiere O, Gamache D, Diguer L, Hérbert E, Larochelle S, Descoteaux J. Personality organization, Five-Factor model, and mental health. J Nerv Ment Dis 2007; 10: 819-29. Disponible en: http:// www.ncbi.nlm.nih.gov/pubmed/18043522 [citado el 6 de abril de 2015].

18. Kernberg O. Trastornos graves de la Personalidad. Colombia: El Manual Moderno; 1992.

19. Carver C, Scheier M. Teorías de la personalidad (3a ed.). México: Pentice Hall Hispanoamérica; 1997.

20. Riquelme R, Oksenberg A, editores. Trastornos de Personalidad hacia una Mirada integral. Santiago de Chile: Sociedad Chilena de Salud Mental; 2003.

21. Caligor E, Kernberg O, Clarkin J. Handbook of Dynamic Psychotherapy for Higher level Personality Patology. Washington D.C. American Psychiatric Publishing, Inc.; 2005.

22. Dalbudak E, Evren C, Aldemir S, Coskun KS, Yıldırım FG, Ugurlu H. Alexithymia and personality in relation to social anxiety among university students. Psychiatry Res 2013; 2: 167-72. Disponible en: doi: 10.1016/j.psychres.2012.11.027 [citado el 6 de abril de 2015].

23. Almonte C, Montt M, editores. Psicopatología infantil y de la adolescencia. Santiago de Chile: Mediterráneo; 2012.

24. Valdivia M, Condeza M. Psiquiatría del adolescente. Santiago de Chile: Mediterráneo; 2006.

25. Montero I, León OG. Sistema de clasificación del método en los informes de investigación en Psicología. Int J Clin Health Psychol 2005; 1: 115-27. Disponible en: http://www.redalyc.org/articulo. oa?id=33701007 [citado el 6 de abril de 2015].

26. Hernández Sampieri R, Fernández C, Baptista
M. Metodología de la Investigación. México: Mc Graw Hill; 2010.

27. Hoffman M. Adaptación y validación para Chile del inventario de organización de la personalidad versión 2001 (Tesis para optar al título profesional de psicólogo). Universidad del Desarrollo, Santiago de Chile; 2004.

28. Ben-Dov P, Martínez C, Núñez C, Ortega P, Oksenberg T, Morales A, et al. El inventario de organización de la personalidad (IPO): sus características y aplicaciones. Ter psicol 2002; 1: 41-51. Disponible en: http://bases.bireme.br/cgi-bin/ wxislind.exe/iah/online/?IsisScript=iah/iah.xis\&s $\mathrm{rc}=$ google $\&$ base $=$ LILACS\&lang $=\mathrm{p} \&$ nextAction $=1$ nk\&exprSearch $=389254 \&$ indexSearch=ID [citado el 6 de abril de 2015].

29. Reyes L, Russo A. Descripción de la organización estructural de la personalidad de los adolescentes que cursaron primer semestre en un programa de psicología de la ciudad de Barranquilla. Psychologia. Avances de la disciplina 2008; 2: 65-91. Disponible en: http://www.redalyc.org/articulo. oa?id=297225162002 [citado el 6 de abril de 2015].

30. Antúnez Z, Vinet EV. Escalas de Depresión, Ansiedad y Estrés (DASS-21): Validación de la Versión Abreviada en Estudiantes Universitarios Chilenos. Ter psicol 2012; 3: 49-55. Disponible en: http:// www.redalyc.org/articulo.oa?id=78524692005 [citado el 6 de abril de 2015].

31. Cova Solar F, Alvial SW, Aro DM, Bonifetti DA, Hernández MM, Rodríguez CC. Problemas de Salud Mental en Estudiantes de la Universidad de Concepción. Ter psicol 2007; 2: 105-12. Disponible en: http://www.redalyc.org/articulo. oa?id=78525201 [citado el 6 de abril de 2015].

32. Saavedra GE, Reynaldos QC. Caracterización cognitiva y emocional de los estudiantes de la Universidad Católica del Maule: años 1999, 2001, 2003. Estudios Pedagógicos 2006; 2: 87-102. Disponible en: http://www.redalyc.org/articulo. oa?id=173514131004 [citado el 6 de abril de 2015].

33. Dávila FA, Ruiz CR, Moncada AL, Gallardo RI. Niveles de ansiedad, depresión y percepción de apoyo social en estudiantes de Odontología de la 
Universidad de Chile. Revista de Psicología 2011; 2: 147-72. Disponible en: http://www.redalyc.org/ articulo.oa?id=26421338007 [citado el 6 de abril de 2015].

34. Lange I, Vio F. Guía para Universidades Saludables y otras Instituciones de Educación Superior. Santiago de Chile: Productora gráfica Andros Ltda; 2006.

35. Quiroga S, Castro A, Fontao N. La Evaluación de la Estructura de la Personalidad: adaptación Argentina del Inventario de Organización de la Per- sonalidad (IPO). Subjetividad y Procesos Cognitivos 2003; 3: 188-219. Disponible en: http://dspace.uces.edu.ar:8180/xmlui/handle/123456789/510 [citado el 6 de abril de 2015].

36. Soutullo C, Mardomingo M. Manual de psiquiatría del niño y del adolescente. Madrid, España: Editorial Médica Panamericana; 2010.

37. Muñoz N, Barraza R, Pérez C, Ortiz L. Repensando la selección de la carrera de Medicina desde los factores que inciden en la formación. Rev Med Chile 2015; en prensa.

\section{Correspondencia:}

René Barraza López

Escuela de Psicología, Facultad de Ciencias

Sociales, Universidad Santo Tomás,

Ruta 5 norte 1068, La Serena.

Teléfono: 56-51-2473223 / 56-51-2473288.

E-mail: renejavierbarraza@santotomas.cl

renebarrazalopez@gmail.com 